\title{
FEUERBACH STATT MARX
}

\author{
ZUR VERFASSERSCHAFT DES AUFSATZES \\ „LUTHER ALS SCHIEDSRICHTER \\ ZWISCHEN STRAUSS UND FEUERBACH"
}

Der Aufsatz „Luther als Schiedsrichter zwischen Strauss und Feuerbach" erschien im zweiten Band der von Arnold Ruge 1843 in der Schweiz herausgegebenen „Anekdota zur neuesten deutschen Philosophie und Publizistik", Seite 206-208, und war gezeichnet mit „Kein Berliner". Rjazanov druckt ihn in der MEGA, I, 1, 1, S. 174-175, ab mit der Bemerkung, die Autorschaft von Marx sei ,als sicher anzunehmen". ${ }^{1}$ Von der MEGA übernehmen den Aufsatz unter anderen die im DietzVerlag erscheinende Ausgabe „Marx-Engels-Werke"2 und die von H. J. Lieber veranstaltete „Karl-Marx-Ausgabe” in der Wissenschaftlichen Buchgesellschaft. ${ }^{3}$ Die von Hermann Becker 1851 herausgegebenen „Gesammelten Aufsätze von Karl Marx" und die von Franz Mehring veranstaltete Ausgabe „Aus dem literarischen Nachlass von K. Marx, Fr. Engels und F. Lassalle" 4 enthalten den Aufsatz nicht.

Für die Marxbiographie kann der kleine Aufsatz die Funktion erfüllen, die Feuerbachrezeption des jungen Marx vor der „Kritik des Hegelschen Staatsrechts" und den ökonomisch-philosophischen Manuskripten $\mathrm{zu}$ dokumentieren. ${ }^{5}$

1 MEGA I, 1, 1, S. XL.

Bd. I, 1957, S. 26-27.

Bd. I, 1962, S. 107-109.

4 Bde, 1902.

Vgl. z.B. jüngst H. J. Lieber und P. Furth in der Lieberschen Ausgabe, Band I, S. 994; H. Gollwitzer, Marxistische Religionskritik und christlicher Glaube (Marxismusstudien, IV, 1962, S. 56) ; A. Cornu, Karl Marx und Friedrich Engels. Leben und Werk. Eine Biographie, Berlin, 1954, I, S. 259, und letztlich W. Schuffenhauer, Feuerbach und der junge Marx, Berlin 1965, vor allem S. 24-27 u.ö. - Schuffenhauer, der das Verhältnis des jungen Marx zu Feuerbach in den Jahren 1841-44 am intensivsten untersucht, bemerkt mit Recht, dass der Verfasser der Schiedsrichterzeilen das gleiche von Feuerbach geübte Verfahren der Zitation „historischer Zeugnisse” und „objektiver Tatsachen” in der empirischen Analyse anwende, übersieht jedoch, dass Feuerbach diese Häufung von Lutherzitaten erst in der 2. Auflage des „Wesens des Christentums” an der er im Jahre 1842 arbeitete, zum ersten Male bringt (a.a.O., S. 24). Schuffenhauers Skizzierung des „gesamten Prozesses” der Marxschen Entwicklung 1841-44 basiert gravierend auf der unüberprüften Voraussetzung der Marxschen Autorschaft an den Schiedsrichterzeilen für den Anfang des Jahres 1842 (a.a.O., S. 26, 153, 190 u.ö.). 
In diesem Aufsatz wird die Verschiedenartigkeit der zwar entmythologisierenden, aber noch theologischen und der psychologischen, die Religion nicht umdeutenden, sondern aufhebenden Religionskritik betont. Auf der Grundlage einiger Lutherzitate wird die Richtigkeit und "natürliche" Selbstverständlichkeit der Feuerbachschen Lösung des Problems der christlichen Entfremdung und metaphysischen Seinsdopplung verteidigt; Strauss, der an die Stelle der alten eine neue Mythologie setze, bleibe noch innerhalb der theologischen Befangenheit, befreie den Menschen nicht zu seiner eigenen natürlichen Wahrheit und Freiheit. Die hier umrissene Position wird in die Entwicklung des jungen Marx um die Jahreswende 1841/42 eingeordnet.

Aus dem Briefwechsel von Marx geht jedoch seine Verfasserschaft an dem Artikel nicht hervor. Am 10.2.1842 schickte Marx die „Bemerkungen über die neueste preussische Zensurinstruktion"1 an Ruge und versprach ihm am 20.3.1842 einen grösseren Beitrag „Über Religion und Kunst mit besonderer Beziehung auf christliche Kunst", ${ }^{2}$ der zunächst als Beitrag zu Bruno Bauers zweitem Teil der "Posaune”, „Hegels Lehre von der Religion und Kunst vom gläubigen Standpunkt aus beurteilt", 3 erscheinen sollte, den Marx dann aber doch nicht an den Verleger sandte, weil er den hinter der Maske eines pietistischen Gläubigen sich versteckenden „Posaunenton” durch eine „freiere, daher gründlichere Darstellung" ersetzen wollte. ${ }^{4}$ Am 27.3.1842 versprach er Ruge ausser dem einen schon übersandten noch vier weitere Aufsätze: „Ich werde Ihnen vier Aufsätze einsenden: 1. ,über christliche Kunst', 2. ,über die Romantiker', 3. ,das philosophische Manifest der historischen Rechtsschule', 54 . die positiven Philosophen"'. ${ }^{6}$ Marx schickte jedoch keine weiteren Beiträge an Ruge, da er im Zusammenhang mit dem Aufsatz über die religiöse Kunst „in allerlei Untersuchungen hineingeraten" ist, „die noch längere Zeit hinnehmen werden". ${ }^{7}$ Ruge mahnte Marx im Juni 1842 und am $7.8 .1842^{8}$ und forderte ihn schliesslich am 21.10.1842, das Manuskript, mit dem offensichtlich das über die christliche Kunst gemeint ist, entweder umgehend an ihn oder gleich nach Zürich zum Druck zu senden, „falls es nur nicht über 4-5 Bogen ist". 9 Trotz umfangreicher Vorstudien kam

1 MEGA I, 1, 2, S. $266 \mathrm{f}$.

MEGA I, 1, 2, S. 272.

3 Vgl. Bauer an Marx am 6.12.1841, MEGA I, 1, 2, S. $263 f$.

1 Marx am 20.3.1842 an Ruge, MEGA I, 1, 2, S. 272.

s Erschienen in der Rheinischen Zeitung am 9.8.1842.

- MEGA I, 1, 2, S. 274.

7 Ebenda.

MEGA I, 1, 2, S. 275 ; $322 f$.

- MEGA I, 1, 2, S. 281. - Falls Marx dann doch noch etwas an Ruge geschickt haben sollte, ist es nicht mehr in die "Anekdota" aufgenommen worden; vgl. 
Marx jedoch nicht zur abschliessenden Ausarbeitung des Aufsatzes, ${ }^{1}$ zunächst wegen seiner Arbeitsüberlastung durch die Redaktionstätigkeit an der Rheinischen Zeitung, dann aber auch weil es ihm darum ging, wie er am 30.11.1842 an Ruge schrieb, „die Religion mehr in der Kritik der politischen Zustände als die politischen Zustände in der Religion zu kritisieren ... da die Religion an sich inhaltslos nicht vom Himmel, sondern von der Erde lebt und mit der Auflösung der verkehrten Realität, deren Theorie sie ist, von selbst stürzt”. ${ }^{2}$ Wichtigstes Resultat dieser Studien dürfte, wie Marx in der Vorrede zur „Kritik der politischen Okonomie”, 1859, in Beziehung auf "die Deutsche Ideologie” schrieb, die „Selbstverständigung” gewesen sein, die hier allerdings noch nicht bis zu dem Punkt gediehen war, der eine literarische Fixierung zuliess. Marx war jedenfalls nicht daran interessiert, sich in die diversen religionskritischen Streitereien der Hegelschule einzumischen, nachdem von ihm einmal der grundlegende Gedanke Feuerbachs rezipiert worden war.

Schon am 2.9.1841 schrieb Moses Hess an Auerbach, Marx gehe „nicht nur über Strauss, sondern auch über Feuerbach heraus”. ${ }^{3}$ Dass Marx spätestens im Frühjahr 1842 dem Feuerbachschen Standpunkt kritisch gegenübertritt, geht aus seiner Mitteilung an Ruge vom 20.3. 1842 hervor, dem er schrieb, dass er in seiner Abhandlung über die christliche Kunst auch über das allgemeine Wesen der Religion spreche, „wo ich einigermassen mit Feuerbach in Kollision gerate, eine Kollision, die nicht das Prinzip, sondern seine Fassung betrifft".4 Bruno Bauer äusserte sich am 12.4 .1842 besorgt, dass Marx durch seine Kritik an Feuerbach diesen als potentiellen Mitarbeiter an der von ihnen geplanten atheistischen Zeitschrift verärgern könne: „wenn Du nun Feuerbach vornimmst, woher dann Leute?"5 Am 13.3.1843 schliesslich umriss Marx seine Feuerbachkritik in Beziehung auf die ihm gerade bekanntgewordenen „Vorläufigen Thesen zur Reformation der Philosophie"6 an der für ihn zentralen Stelle: „Feuerbachs Aphorismen sind mir nur in dem Punkt nicht recht, dass er zu sehr auf die Natur und zu wenig auf die Politik hinweist. Das ist aber das einzige

M. Fleischer an G. Jung am 16.12.1842 (Rheinische Briefe und Akten zur Geschichte der politischen Bewegung 1830-1850, ges.u.hg. J. Hansen, Bd. I, [18301845], Essen 1919, S. 386).

1 Zu den Vorstudien vgl. die Exzerpthefte, MEGA I, 1, 2, S. 114-118, und M. Rubel, Les Cahiers d'Etudes de Karl Marx (International Review of Social History, II, 1957, S. 395f.).

2 MEGA I, 1, 2, S. 286; vgl. manche Gedankengänge in MEGA I, 1, 1, S. 232-250.

MEGA I, 1, 2, S. 261.

4 MEGA I, 1, 2, S. 272.

5 MEGA I, 1, 2, S. 253.

- Anekdota, II, S. 62-86. 
Bündnis, wodurch die jetzige Philosophie eine Wahrheit werden kann."1 Weder in seinen Schriften noch in seinem Briefwechsel lässt sich ein besonderes Interesse an den religionskritischen Streitigkeiten der verschiedenen Lager feststellen. Ihm genügt die grundsätzliche Erkenntnis Feuerbachs, die für ihn die Grundlage zu weiterem, über Feuerbach hinausgehendem, jetzt politischem Denken und Handeln ist. Eine Verteidigung der Feurbachschen Position gegenüber dem Straussschen Standpunkt hätte sicher einen Hinweis auf die Bedingtheit auch der Feuerbachschen Konsequenzen und auf die Notwendigkeit, diese zu überwinden, enthalten. Die in dem Aufsatz „Luther als Schiedsrichter zwischen Strauss und Feuerbach" vorgelegten Lutherzitate wären zudem die einzigen Lutherzitate, die Marx bringt, noch dazu aus einer Ausgabe, die hinter der viel gebräuchlicheren von Walch ${ }^{2}$ oder der damals neu erscheinenden Erlanger Ausgabe ${ }^{3}$ an Verbreitung zurückstand. Für den jungen Marx hat zudem immer die antike Religionskritik mehr bedeutet, als es eine indirekte Bestätigung der Feuerbachschen Thesen durch den Glaubensstandpunkt Luthers hätte tun können. ${ }^{4}$ Marx hielt schon im Jahre 1842 den Standpunkt der blossen Religionskritik für überholt und forderte, diese mit der Kritik der Gesellschaft und Politik zu vertauschen. - In dem Briefwechsel zwischen Marx und Feuerbach 1843/44, den Schuffenhauer 1965 erstmalig vollständig edierte $^{5}$ und in dem Marx als der um Mitarbeit an den Deutsch-Französischen Jahrbüchern Werbende auftritt, ${ }^{6}$ hätte dieser, falls er der Autor gewesen wäre, sicherlich nicht versäumt, den Aufsatz „Luther als Schiedsrichter zwischen Strauss und Feuerbach" bei Feuerbach in Erwähnung zu bringen.

Aus einem jetzt erstmalig veröffentlichten Brief Feuerbachs an Ruge vom 15.2.1842 geht hervor, dass Feuerbach in der Anlage ein paar „flüchtige Zeilen" über Strauss und sich mitschickte. ${ }^{7}$ Feuerbach ar-

1 MEGA I, 1, 2, S. 308.

24 Bde, Halle 1740-1753.

${ }^{3}$ Erschienen 1826-1857 u. 1829-1886.

4 Vgl. die Liebersche Ausgabe, Bd. I, S. 77-106; dazu MEGA I, 1, 114-118.

${ }^{5}$ Schuffenhauer, a.a.O., S. 194-218.

6 Vgl. auch den Brief vom 11.8.1844, ebenda S. 207-214.

7 Vgl. Ludwig Feuerbach, Sämtliche Werke, Bd. XIII, hg. H. M. Sass, 1964, S. 390. Der Wortlaut des ersten Absatzes lautet: „Teurer Freund! Sie haben mich durch Thre ausserordentliche Güte wahrhaft gerührt. Empfangen Sie dafür einstweilen meinen herzlichen Dank. Ich werde die Bücher Ihnen natürlich in derselben eleganten Beschaffenheit wieder zustellen. Ich kann zunächst nur flüchtige Blicke hineinwerfen. Ich habe Folianten aus den Erlanger und Nürnberger Bibliotheken, und die ersteren muss ich in einigen Wochen wieder zurückschicken. Soviel habe ich aber gesehen, dass der Strauss nur die hölzernen Schulbegriffe imputiert. Es lohnt sich gar nicht der Mühe, darüber zu streiten. 
beitete zu dieser Zeit an der zweiten Auflage des „Wesens des Christentums". Die ihm von Ruge zugesandten Bücher konnte er nur flüchtig zur Kenntnis nehmen; dabei mag ihn am meisten der zweite Band von D. Fr. Strauss' „Die christliche Glaubenslehre in ihrer geschichtlichen Entwicklung und in ihrem Kampf mit der modernen Wissenschaft", der 1841 erschien, interessiert haben. Für Feuerbach hatte Strauss die Theologie noch nicht überwunden. Der Strausssche Standpunkt war so weit von seinem eigenen entfernt, dass es sich nicht einmal lohnte, mit Strauss in einen Streit einzutreten. Und doch griff Feuerbach zur Feder und schickte Ruge "flüchtige Zeilen über Strauss und mich". Es blieb Ruge überlassen, ob er diese Zeilen in seine eigene Feuerbachrezension einbaute oder ob er sie separat veröffentlichte. ${ }^{2}$

Da es in dem Gegensatz zwischen Strauss und Feuerbach um die grundsätzliche Position beider ging und Feuerbach es ablehnte, sich mit Strauss um Einzelheiten zu "streiten", konnte es in dem kurzen Beitrag nur um das paradigmatische - polemische oder auch nicht polemische - Aufzeigen des grundsätzlichen Gegensatzes gehen. An einer solchen Klärung allerdings musste Feuerbach sehr gelegen sein. Es lag für Feuerbach nahe, diesen Unterschied durch einen Hinweis auf Äusserungen Luthers deutlich zu machen, der für ihn "ein ganzer Mann"3 und der Idealtyp eines christlichen Gläubigen war, insofern er

Die flüchtigen Zeilen über Strauss und mich überlasse ich ganz Ihrem eigenen Ermessen, ob Sie sie wollen drucken lassen oder nicht, oder nur in ihrer eigenen Rezension über mich davon irgendeinen Gebrauch machen wollen, wenn noch Zeit dazu". S. dazu auch unten S. 119.

1 Ebenda.

2 Die Rugesche Rezension erschien, weil von der Zensur nicht zum Erscheinen in den Deutschen Jahrbüchern zugelassen, in den Anekdota, II, S. 3-61. Ruge nimmt in ihr den gleichen Standpunkt ein, wie der Verfasser, der Luther als Schiedsrichter zwischen Strauss und Feuerbach anruft; Strauss habe nur theoretisches Interesse an der Religion, Feuerbach praktisches, Strauss kritisiere metaphysisch, Feuerbach aber psychologisch, anthropologisch; vgl. a.a.O., S. 16f. - Feuerbachs Zeilen konnten in Ruges Rezension nicht mehr eingebaut werden, weil diese bereits fertig war und, wie Ruge am 24.2.1842 an Feuerbach schrieb, auch schon von der Zensur gestrichen war - ebenso wie Feuerbachs „Vorläufige Thesen zur Reformation der Philosophie" -; vgl. Feuerbach, S.W., a.a.O., S. 93f., und unten S. 119.

- Luther entscheidet sich ebenso eindeutig und radikal "für Gott wider den Menschen", wie Feuerbach sich gegen Gott für den Menschen entscheidet (Das Wesen des Glaubens im Sinne Luthers, 1844, in: Sämtliche Werke, hg. L.F., Bd. I, 1846, S. 267). - Wilh. Bolin, Feuerbachs Biograph, stellt ausdrücklich Feuerbachs Vorliebe für Luther, dem er sich verwandt fühlte, fest; vgl. Über Ludwig Feuerbachs Briefwechsel und Nachlass. Zur Verteilung an die Freunde des Verstorbenen als Privatmitteilung des Verfassers gedruckt, Helsingfors 1877 , 47 S. : ,., Ich bin Luther II', pflegte Feuerbach scherzend zu sagen”, a.a.O., S. 43. - Alle gesperrten Stellen der Lutherzitate im "Wesen des Christentums” sind von Feuerbach hervorgehoben; die Zedlersche Ausgabe kennt keine Sperrungen - 
in seinen Schriften wesentlich unphilosophisch und untheologisch rein aus seinem Christsein heraus spricht und argumentiert. ${ }^{1}$ Luther war überhaupt die grosse Entdeckung Feuerbachs in den ersten Monaten des Jahres 1842. Die zweite Auflage des "Wesens des Christentums" unterscheidet sich wesentlich dadurch von der ersten, dass Feuerbach in ihr seine Thesen durch eine auffallend grosse Zahl von Lutherzitaten zu stützen sucht. ${ }^{2}$ Im Zusammenhang mit den Arbeiten an der zweiten

auch die Sperrungen in „Luther als Schiedsrichter zwischen Strauss und Feuerbach" sind von dem Verfasser dieses Aufsatzes vorgenommen worden! - Im Feuerbachnachlass, der in der Münchener Universitätsbibliothek aufbewahrt wird, findet sich in den Exzerptheften Feuerbachs aus der Lutherausgabe Zedlers folgende Stelle: „An G[ott] soll man keck s[ein] u[nd] nicht verzagen. Denn was ich $u[n d]$ andere $M$ [enschen] nicht können $u[n d]$ vermögen, das kann $u[n d]$ vermag er. Kann ich $u[n d]$ andere Leute mehr nicht helfen, so kann er mir helfen $\mathrm{u}$ [nd] mich auch v[om] Tode erretten, wie Psalm 68 s[iehe] p[agina] 443 I Pred[igt] a[m] 16. S[onntag] $\mathbf{n}$ [ach] d[em] S[onntag] Trinitatis Luc. 7. In G[ott] $\mathrm{u}$ [nd] $\mathrm{s}$ [ein] Sohn sollen wir keck $\mathrm{s}$ [ein] denn was wir nicht können, das kann er, was wir nicht haben, das hat er. Können wir nicht helfen, so kann er helfen u[nd] will es sehr gern u[nd] willig thun ibid. 445". Am Rande zu diesem Exzerpt bemerkt Feuerbach "Trost der Allmacht im Unglück”. Im „Wesen des Christentums" und in den Lutherstudien finden sich zwar die anderen von Feuerbach exzerpierten Stellen aus Luther, nicht jedoch die in den Schiedsrichterzeilen verwandten.

1 Mitte Januar 1842 schrieb Feuerbach an seinen Verleger Wigand, er habe das "Wesen des Christentums" für die zweite Auflage bereits so weit umgestaltet, dass es „den Rang einer unumstösslichen evidenten Wahrheit, einer wissenschaftlichen, sogar mehr als wissenschaftlichen - einer welthistorischen Tatsache bekommen muss. Auch habe ich die köstlichsten Belegstellen - zumal aus Luther und Augustin den beiden Matadoren des Christentums - bereits gesammelt"; S.W., Bd. XII, 1964, S. 83.

Während die erste Auflage keine Zitate aus der Zedlerschen Lutherausgabe, 1729-1740, bringt, enthält allein der Anhang, der in der ersten Auflage unter dem Titel "Anmerkungen und Beweisstellen”, dort S. 381-450, nur ein Lutherzitat aus dem grossen Katechismus bringt, in der zweiten Auflage unter dem Titel „Erläuterungen, Bemerkungen, Belegstellen”, dort S. 414-522, etwa 100 Zitate aus der Zedlerschen Ausgabe, davon fast jedes fünfte aus dem XVI. Band. Die Bedeutung, die das Studium Luthers für die zweite und die weiteren Auflagen des „Wesens des Christentums" und Feuerbachs tiefere Durchdringung des gesamten religionsgeschichtlichen Materials hat, ist bisher nicht beachtet worden. Vgl. z.B. S. Rawidowicz, Ludwig Feuerbachs Philosophie, 1931 (Neudruck 1964), S. 82f; auch S. 160-163. Die ",kritische Ausgabe” von K. Quenzel, 1904 bei Reclam (Bibl. Nr. 4571-75), legt die dritte Auflage von 1849 seiner Ausgabe zu Grunde, nimmt aber, ,da sie als Volksausgabe gedacht ist”, von einer Mitteilung der Lesarten Abstand" (a.a.O., Vorwort, S. 17). Der von W. Schuffenhauer in seiner zweibändigen Ausgabe des „Wesens des Christentums”, 1956 im Akademie-Verlag, angestellte und an anderen Stellen zuverlässige Textvergleich der auch von ihm zu Grunde gelegten dritten Auflage mit den beiden vorhergehenden lässt bedauerlicherweise die Änderungen in den Anmerkungen unberücksichtigt und bringt von den Änderungen in dem Anhang ,Erläuterungen, Bemerkungen, Belegstellen" nichts als die Mitteilung von vier lapidaren Druckfehlern in der dritten Auflage. S. auch unten S. 119. 
Auflage des „Wesens des Christentums” dürften auch die 1844 und 1846 veröffentlichten "Luther-Studien" entstanden sein. ${ }^{1}$ Hier arbeitete er seine Deutung der religiösen Phänomene dadurch plastisch heraus, dass er geschickt und klug ausgewählte längere und kürzere Zitate Luthers knapp kommentiert. ${ }^{2}$ Luther wird hier als Kronzeuge und kirchenväterliche Autorität für die „Richtigkeit” der Feuerbachschen Thesen, in den "Anekdota" als Schiedsrichter zwischen der „richtigen” Sicht Feuerbachs und derjenigen eines noch in den Schulbegriffen der Theologie befangenen "Theologen" zitiert. $^{3}$

In der Knappheit, in der das Problem in den Anekdota abgehandelt wird, büssen die Lutherzitate allerdings manches von ihrer Wirkung ein, da die von ihnen geforderte Aussage nur demjenigen deutlich ist, der sie schon vom Feuerbachschen Standpunkt her liest und wertet, wie ja überhaupt die Feuerbachsche Religionskritik nicht die Frage einer von anderen theologischen oder philosophischen Interpretationen sich mehr oder weniger unterscheidenden Auslegung der Religion ist, sondern die einer grundsätzlich anderen Perspektive. Die angeführten Lutherzitate, für sich genommen, können genau so gut vom Straussschen Standpunkt her interpretiert werden. Jemandem jedoch, der einmal die religiösen Phänomene aus einer bestimmten Sicht zu sehen sich gewöhnt hat, erscheinen alle weiteren gesichteten Zeugnisse als Beweis des eigenen perspektivischen Standpunktes. ${ }^{4}$ Dass eine das gesamte religiöse Leben uminterpretierende These nicht durch die Interpretation immer neuen Quellenmaterials bewiesen wird - ein Verfahren, das Feuerbach vom „Wesen des Christentums" an immer wiederholt - ist dem Verbreiter einer solchen These in Bezug auf sein eigenes Tun allerdings nicht in jedem Falle einsichtig; das Gleiche gilt von der Überzeugungskraft von Zitaten, wenn sie mit dem Ziel interpretiert werden, den Ansatzpunkt eben dieser Interpretation zu be-

1 Vgl. Feuerbachs Briefe an Chr. Kapp vom 13.5.1844 und an Fr. Kapp vom 15.10.1844, S.W., Bd. XIII, 1964, S. 135f. und $137 f$.

2 In dem Begleitbrief, mit dem er das Manuskript an den Verleger übersendet, heisst es : „Die Schrift enthält - was ich sagen kann, ohne mich einer lächerlichen Arroganz schuldig zu machen - das Tiefste, was je gesagt worden über das Wesen Luthers als eines Mannes, dessen die heutigen Deutschen nicht mehr wert sind."; S.W., Bd. XII, 1964, S. 108.

3 Es heisst dort: „Luther - eine sehr gute Autorität, eine Autorität, die alle protestantischen Dogmatiken samt und sonders unendlich überwiegt, weil die Religion bei ihm eine unmittelbare Wahrheit, sozusagen Natur war - Luther entscheide"; Anekdota, II, S. 206.

"Feuerbach meint das Gleiche, wenn er im Vorwort zur zweiten Auflage des "Wesens des Christentums" erkennt: „,wer einen Glaubensartikel umstösst, der stösst, wie schon Luther sagte (Leipziger Ausg., 1731, T. XI, p. 426) alle um"; 2. Aufl., 1843, S.V. 
weisen. ${ }^{1}$ Dieses von Feuerbach her geläufige Verfahren verwendet auch der Autor, der Luther als Schiedsrichter zwischen Strauss und Feuerbach anruft.

Kurz: Feuerbach hat am 15.2.1842 einige kurze Bemerkungen über Strauss und sich an Ruge geschickt. Es liegt nahe, dass er dabei versuchte, seine Position durch die Anrufung Luthers als eines Schiedsrichters $z u$ verteidigen.

Die bisherige Einordnung des Aufsatzes „Luther als Schiedsrichter zwischen Strauss und Feuerbach" in das Werk von Marx brachte die Herausgeber vor das Problem der Datierung seines Entstehens. Den Beitrag, wie es sich aus dem Schriftwechsel zwischen Marx und Ruge ergeben hätte, nach dem 21.10.1842 zu datieren, konnten sich die Herausgeber nicht entschliessen wegen der vielfältigen Äusserungen Marxens zu Feuerbach und zu religionskritischen Fragen im Laufe des Jahres 1842, die einen kritischeren Ton gegenüber Feuerbach für notwendig halten.

Rjazanov verfällt daher auf den Ausweg, den Aufsatz auf Anfang $1842 \mathrm{zu}$ datieren als Antwort auf die in den Deutschen Jahrbüchern am 1.11.1841 und am 10. und 11.1.1842 erschienenen religionskritischen Artikel, die mit den Zeichen „Ein Berliner”, „Auch ein Berliner” und „Ein Philosoph" gezeichnet waren. Im November oder Dezember erst des Jahres 1842 hätte dann Marx trotz seines jetzt gegenüber Feuerbach geänderten Standpunktes das Manuskript unbearbeitet an Ruge oder den Drucker gesandt - und das, obwohl er andere grössere Beiträge religionskritischen Inhalts, weil sie seinen neugewonnenen Standpunkt nicht widerspiegeln konnten, zurückhielt oder sogar vernichtete.

Bei einer Einordnung des Beitrages in das Werk Feuerbachs würde sich das bekannte Feuerbachbild nicht ändern. Seine Stellung zu Strauss, seine Wertschätzung Luthers und seine literarische Form, mit Zitaten zu arbeiten und das Du des Lesers unmittelbar anzusprechen, würden bestätigt werden. Von einem besonderen Reiz wäre allerdings die Tatsache, dass das Wortspiel von dem Feuer - bach - „der Feuerbach ist das Purgatorium der Gegenwart” - von ihm selbst in die Diskussion gebracht worden wäre. ${ }^{2}$

${ }^{1}$ Ruge bemerkt bereits im Jahre 1842 an Fleischer 14.5.1842), Feuerbach schreibe "selten und nur in einem Genre"; A. Ruges Briefwechsel und Tagebuchblätter, hg. P. Nerrlich, Bd. I, 1886, S. 275.

2 Im Feuerbachnachlass der Universität München (Cod. ms. 935b, 10) findet sich im Brief eines Dr. Chronik aus Chikago, Illinois, vom 11.11.1869 die Stelle: „Mögen Sie noch lange lange rüstig ein Feuerbach sein, der den Flammen unseres Geistes Revolutionsahnung zuführt, nachdem er einer der Ersten war, der sie angefacht!" 
Wenn man Feuerbach als Verfasser annimmt, so steht auf Grund seines Briefes vom 15.2.1842 fest, dass er sich in seiner Replik unmittelbar auf das Werk von Strauss selbst bezog, jedoch an den letzten der obengenannten Beiträge in den Deutschen Jahrbüchern anknüpfte. Ruge schickte ihm in gewissen Abständen die neuesten Nummern der Jahrbücher; so wird auch die am 15.2.1842 bestätigte Sendung die gerade letzten Nummern enthalten haben. Die Unterschrift könnte sowohl von Feuerbach als auch von Ruge stammen. Ruge hätte den Aufsatz zusammen mit seinem von der Zensur gestrichenen Artikel aufbewahrt, ohne den Versuch zu machen, ihn in den Jahrbïchern abzudrucken, bis er ihn schliesslich, sicher nicht nur aus dem Grund, dass er keine weiteren Beiträge erhielt, in die Anekdota aufnahm.

Es wird also Rjazanov in dem Punkt, dass der Verfasser, wenn er mit „Kein Berliner” zeichnet, sich auf einen der in den Deutschen Jahrbüchern erschienenen oben genannten Artikel beziehen könnte, nicht widersprochen. ${ }^{1}$ Doch kann Rjazanov nicht zugestimmt werden, wenn er davon spricht, dass der Verfasser den Ton der „Posaune” anwende, "durch eine orthodoxe Autorität radikale Wahrheiten zu beweisen", und am Schluss des Artikels selbst in den „Posaunenstil” verfalle. ${ }^{2}$ Der Stil des vorliegenden Aufsatzes unterscheidet sich von dem der „Posaune" dadurch, dass dort der orthodoxen Autorität in scheinheilig frommer Interpretation radikale Meinungen unterschoben werden, während hier die orthodoxe Autorität in ihrem Selbstverständnis als Zeuge für die Richtigkeit einer sie interpretierenden Kritik beispielhaft vorgeführt wird. Letzteres Verfahren ist das bei der Zitation von Autoritäten christlicher Provenienz durch Feuerbach stets angewandte. Was den „Posaunenstil" betrifft, so dürfte sich auch dieser in seiner polemischen und pathetischen Verstiegenheit von der hier sich an das persönliche Du des Lesers wendenden Form unterscheiden, einer Form, die Feuerbach von den "Gedanken über Tod und Unsterblichkeit” an über den „Pierre Bayle” und den „Schriftsteller und der Mensch” bis in seine letzten Schriften hinein kultivierte als die praktische Konsequenz seiner philosophischen Haltung, die er theoretisch z.B. 1843 in den "Grundsätzen der Philosophie der Zukunft” so formuliert: „Die wahre Dialektik ist kein Monolog des einsamen Denkers mit sich selbst, sie ist ein Dialog zwischen Ich und Du."3 Für Marx ist ein solcher Stil nicht typisch.

Die Autorschaft Feuerbachs wäre mit grösster Sicherheit zu be-

\footnotetext{
1 Für den Gegensatz zwischen Strauss und Feuerbach kommt allerdings nur die letzte der obengenannten Kritiken, die mit "Ein Philosoph" gezeichnet ist, in Frage; vgl. Dt. Jbb. vom 11. u. 12.1.1842, S. 30-35.

2 MEGA I, 1, 1, S. XLI.

3 Sämtliche Werke, hg. L. Feuerbach, Bd. II, 1846, S. 345.
} 
haupten, wenn nicht Ruge am 8.3.1843 an Marx schreiben würde, dass dieser demnächst das Honorar für " 2 Aufsätze in den Anekdotis $2 \mathrm{~B}$ [ogen] 3 S[eiten]" bekommen würde. ${ }^{1}$ Nur die Autorschaft Marxens an einem Aufsatz, an dem über die Zensurinstruktion, ist nach dem Brief von Marx an Ruge vom 10.2.1842 und Ruges Antwort vom 25.2.1842 gesichert. ${ }^{2}$ Von den andern nicht mit Namen gezeichneten Artikeln in den Anekdota ist der über „Die Rheinische Zeitung über Pressfreiheit" von Ruge; ${ }^{3}$ der Aufsatz „Die philosophische Kritik und die Deutschen Jahrbücher. Brief eines Rheinländers aus Berlin" dürfte dem Inhalt nach von einem der Bauerschüler in Berlin verfasst sein, nicht jedoch von Marx, der statt der dort geforderten theoretischen und philosophischen Diskussion politische Konsequenzen gefordert hätte; der Aufsatz "Stimmen aus Preussen an Preussen. v. Rhein", von dem Ruge angibt, dass er von dem Zensor der Deutschen Jahrbïcher nicht zum Drucke zugelassen worden sei, ist, wie schon der Stil ausweist, von dem gleichen Verfasser, der in den Deutschen Jahrbüchern 1841 unter dem Titel „Preussen und der Katholizismus" schrieb und mit „v. Rheine” zeichnete. ${ }^{4}$ Für diese Aufsätze kommt eine Verfasserschaft von Marx nicht in Frage.

Der von Ruge angegebene Umfang von zwei Bogen und drei Seiten würde im übrigen garnicht zutreffen, wenn die Seitenzahlen des Marxschen Aufsatzes über die Zensurinstruktion mit denen des Aufsatzes über „Luther als Schiedsrichter zwischen Strauss und Feuerbach" addiert würden; das ergäbe vielmehr die Zahl von 36 Seiten, das heisst von zwei Bogen und vier Seiten. Es gibt überhaupt nur einen Aufsatz in den Anekdota, der die durch die Rugesche Angabe erforderliche Anzahl von zwei Seiten hat: der von Ruge über „Die Rheinische Zeitung über die Pressfreiheit", ${ }^{5}$ der sich in den Anekdota unmittelbar an den Aufsatz über die Zensurinstruktion anschliesst und zu zwei Dritteln aus Zitaten aus dem Marxschen Bericht über die Debatten des Rheinischen Landtages über Pressefreiheit in der Rheinischen Zeitung besteht.

Nun ist es durchaus nicht abwegig, anzunehmen, dass Ruge das Honorar für diesen kurzen Hinweis als Herausgeber und als Autor dem eigentlichen Urheber dieser treffenden und klugen Bemerkungen über Pressefreiheit und Pressegesetz zukommen liess, der dieses Problem

1 MEGA I, 1, 2, S. 306.

MEGA I, 1, 2, S. 267

3 Nach seinem Brief vom Juni 1842 an Marx, MEGA I, 1, 2, S. 275f. Die Autorschaft Ruges ergibt sich aber auch aus dem Inhalt.

- Dt. Jbb., 1841, S. 313-414.

5 Anekdota, I, S. 91-92.

- Rheinische Zeitung vom 12.5.1842, Beiblatt; vgl. in der Lieberschen Ausgabe, Bd. I, S. 139 und 147f. Uber die Vorgeschichte dieses kurzen Hinweises vgl. Ruge an Marx im Juni 1842, MEGA I, 1, 2, S. 276. 
„auf eine wesentlich neue und schlagend richtige Basis stellt”, und die daher ,wo von Pressfreiheit in Zukunft die Rede ist, wenigstens seinem Prinzipe nach gekannt und zum Grunde gelegt zu werden verdient". ${ }^{1}$ Eine solche Annahme wird gestützt durch die Tatsache, dass Ruge zu dieser Zeit grössten Wert auf eine gute Zusammenarbeit mit Marx legte, mit dem er nur wenig später gemeinsam in Paris die DeutschFranzösischen Jahrbücher herausgab. ${ }^{2}$ Hier bestand für ihn die Möglichkeit in einer kleinen Geste seinen guten Willen zu der in Aussicht genommenen Partnerschaft und Solidarität auch im Okonomischen vorweg $\mathrm{zu}$ dokumentieren. Falls das Honorar wirklich genau nach Seitenzahlen abgerechnet wurde, hatte sich Ruge bereits an den "leeren" Seiten der Zwischentitel ${ }^{3}$ schadlos gehalten. ${ }^{4}$

Aus dem Dargelegten ergibt sich deutlich, welche Schwierigkeiten und Widersprüche mit Rjazanovs Annahme einer Autorschaft Marxens verbunden sind, wenn die Feuerbachkritik von Marx im Jahre 1842 und die Briefwechsel zwischen Marx und-Ruge und Marx und Feuerbach in den Jahren 1841-44 gebührend berücksichtigt werden.

Es liegt daher sehr nahe, Feuerbach die Verfasserschaft zuzusprechen, die sich aus seinem Brief vom 15.2.1842, seinen Lutherstudien und -exzerpten im Frühjahr 1842, seinen stilistischen und schriftstellerischen Eigenheiten und seinem genau in dieser Richtung liegenden Engagement an der Sache nachweisen lässt. Das von Ruge in seinem Brief vom 8.3.1843 gemeinte Honorar bezieht sich auf Marx' Aufsatz über die Zensurinstruktion und seine von Ruge zitierten prinzipiellen Gedanken über das Pressegesetz aus der Rheinischen Zeitung vom 12.5.1842.

Die Annahme einer Verfasserschaft Feuerbachs wird durch gewichtige Argumente gestützt. Sie befreit zudem das auch aus anderen Gründen nicht gerade sehr durchsichtige Bild der Entwicklung des jungen Marx von unnötigem Ballast, indem nun zur Frage der Marxschen Überwindung des feuerbachschen Standpunktes im Jahre 1842 allein noch die Marxschen brieflichen Äusserungen zu Feuerbach vor-

\footnotetext{
1 Anekdota, I, S. 91.

2 Vgl. den Briefwechsel zwischen Ruge und Marx, MEGA I, 1, 2, S. 295-321.

3 Insgesamt 12 Seiten, darunter auch die Seiten 89-90, die zwischen dem Marxschen Aufsatz über die Zensurinstruktion und dem Rugeschen Hinweis auf Marx' Ausführungen in der Rheinischen Zeitung zur Frage der Pressefreiheit stehen.

4 Die Möglichkeit jedoch, dass Ruge in seinem Brief vom 8.3.1843 ein Irrtum unterlaufen ist, der auf seinen ausschliesslich schriftlichen Verkehr mit dem in der Schweiz befindlichen Froebel oder auf seine eigene ruhelose Existenz gerade wieder in dieser Zeit oder einfach auf einen Zufall zurückgeführt werden könnte, ist, wenn nicht sonderlich wahrscheinlich, so doch nicht grundsätzlich auszuschliessen.
} 
liegen, die dokumentieren, dass die Marxsche Kritik an Feuerbach schon $1842 \mathrm{im}$ Grundsätzlichen in die Richtung ging, die durch die 1888 von Engels veröffentlichten Marxschen „Thesen über Feuerbach” formuliert ist.

Während der Drucklegung dieses Aufsatzes habe ich drei weitere Sachverhalte notiert, die die vorgetragene These stützen.

1. Feuerbach hatte am 9.10.1842 (Sämtliche Werke, 13, S. 78ff.) in einem Brief an Christian Kapp diesem ebenso Zitatenmunition geliefert wie jetzt Ruge. An Kapp schickte er eine ganze Serie von Böhme-Zitaten, die zeigen sollten, dass Schelling nichts als ein Böhmeplagiator sei. Er schreibt nach der Aufzählung der Zitate aus Böhme: „Mögen diese Stellen genügen! Es ist diese Stellensucherei eine Mühseligkeit, Geistlosigkeit, dass man aus der Haut fahren, vor Ungeduld verzappeln möchte. Nur Dir zuliebe konnte ich sie übernehmen. Die exzerpierten Stellen und Seitenangaben führen nur als Beispiele an, zur Rechtfertigung der Behauptung, dass der wesentliche Inhalt dieser Schellingschen Schrift Jakob Böhme angehört. Und zu diesem Zweck hast Du genug in Händen. Mehr wäre geistlos."

2. Enrico Rambaldi, Mailand hat entdeckt, dass Ruge bereits schon einmal aus einem Brief von Feuerbach eine kleine Miszelle gemacht hat, hier allerdings in ausdrücklicher Berufung auf einen Brief und eine Zitation des Briefes, der aus gerade diesem Grunde nicht im Rugeschen Nachlass vorliegt, weil er mit Ruges Bemerkungen zum Setzer wanderte. Im Wastebook der Halleschen Jahrbücher von 1840, Sp. 93 und 94 berichtet Ruge unter dem Titel „Das Pathos der Kritik und die Kritik der unreinen Vernunft" von der Ankündigung des Briefschreibers, ein neues Opus betreffend (gemeint ist „Das Wesen des Christentums”) :Ein junger Gelehrter, dessen Bedeutung in den ernsten Konflikten der heuchlerischen Theologie mit der gesunden Wissenschaft in kurzem noch energischer, als bisher, hervortreten wird, schreibt an einem Werke, welches er scherzhaft eine Kritik der unreinen Vernunft nennt, die ihm eben so sehr am Herzen liegt, als ihre Inhaber den herzlichen Anteil bloss vorgeben. Er spricht sich darüber brieflich folgendermassen aus: [Es folgt die Briefstelle, dann schliesst Ruge] Wir brauchen den geistreichen und tapferen Verfasser nicht zu nennen. Er ist seinen Feinden und Freunden gleich kenntlich. Seinem Buche aber sehen wir mit Verlangen entgegen; er wird sein Publikum nach beiden Seiten hin beträchtlich vermehren und die grosse Aufgabe der Wiederherstellung eines Gewissens in der Wissenschaft, d.h. eine neue Reformation wesentlich befördern!"

3. Carlo Ascheri, Heidelberg, weist mich auf den Brief Feuerbachs an seinen Bruder Friedrich vom 10.12.1843 hin (Feuerbachnachlass der Münchener Universitätsbibliothek: $4^{\circ}$ Cod. ms. 935a, $12(16)$ ), in dem Feuerbach schreibt, dass er 21 Bände der Leipziger Lutherausgabe selbst besitze „der Gelehrten Pedante willen", jedoch den von de Wette herausgegebenen Briefwechsel und die neueren Lutherausgaben aus Erlanger und Nürnberger Bibliotheken noch einsehen wolle. 